\title{
Aciertos y falencias de los modelos analógicos asociados a terremotos utilizados en enseñanza
}

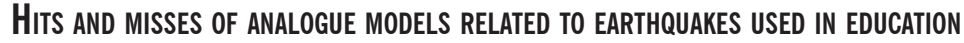 \\ ACERTOS E DEFICIENCCIAS DOS MODELOS ANALÓGICOS ASSOCIADOS A TERREMOTOS USADOS NO ENSINO \\ José Selés-Martínez \\ Doctor em Ciencias (Geolocía) pela Universidad de Buenos Aires. Especialsta em Hidrogelogía pela Universidad Complutense de Madrid.
}

Abstract: The theoretical foundations of the relationships between force, stress and deformation, and also on the nature and propagation of waves are briefly developed. Simple experiences, which are carried out with common low-cost materials, but which serve to carry out didactically appropriate practical activities, have been intentionally selected. The models are analyzed from the perspective of their scope, their different levels of depth and, above all, their limitations, taking into account that this is the least approached subject in usual analyzes, no matter it may induce misconceptions or oversimplification among students. This study opens new perspectives in the use of traditional teaching experiences on earthquakes by incorporating a new dimension: the analysis of the models, their results and the associated technical concepts, what is considered to be key for the students' understanding of the methods of action and reflection used in scientific research.

Resumen: Se exponen brevemente los fundamentos teóricos de las relaciones entre fuerza, esfuerzo y deformación y sobre la naturaleza y propagación de ondas, empleándose experiencias sencillas con materiales simples y de bajo costo, pero que sirven para realizar actividades prácticas didácticamente adecuadas. Los modelos se examinan desde la perspectiva de su alcance, sus diferentes niveles de profundidad y, sobre todo, de sus limitaciones, considerando que éste es el aspecto menos abordado en los análisis habituales a pesar de que puede inducir falsas concepciones o simplificaciones excesivas con respecto al fenómeno real. Se considera que este estudio abre nuevas posibilidades para abordar experiencias tradicionales de enseñanza sobre terremotos al incorporar una nueva dimensión: el análisis de los modelos usados, sus resultados y los conceptos teóricos asociados. De este modo se brindaría a los estudiantes una aproximación a los métodos de acción y reflexión utilizados en la investigación científica.

Resumo: Expõem-se brevemente os fundamentos teóricos das relações entre força, esforço e deformação, bem como sobre natureza e propagação de ondas a partir de experimentos simples e rápidos, que empregam materiais simples e de baixo custo, bastante úteis para atividades didáticas. Examinam-se os modelos quanto a: alcance; diferentes níveis de profundidade e, principalmente, quanto às limitações. Este é 0 aspecto menos abordado nas análises, apesar do potencial de induzir falsas concepções ou simplificações excessivas, em quem aprende, quanto ao fenômeno real. 0 estudo abre novas possibilidades para abordar experiências tradicionais de ensino sobre terremotos, ao incorporar a nova dimensão da análise de modelos usados, seus resultados e conceitos teóricos associados, permitindo aproximar os estudantes dos métodos de ação e reflexão utilizados na investigação científica.
Citation/Citação: Sellés-Martínez, J. (2020). Aciertos y falencias de los modelos analógicos asociados a terremotos utilizados en enseñanza. Terræ Didatica, 16, 1-11, e020029. doi: 10.20396/ td.v16i0.8658592

Keywords: Earth Sciences. Seismic Teaching. Tsunamis. Natural hazards.

Palavras-chave: Ciências da Terra. Ensino de sísmica. Tsunamis. Riscos naturais.

Palabras clave: Ciencias de la Tierra, Enseñanza de sísmica, Tsunamis, Riesgos naturales.

Manuscript/Manuscrito:

Received/Recebido: 20/03/2020

Revised/Corrigido: 11/06/2020

Accepted/Aceito: 20/06/2020

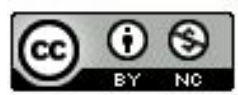

\section{Introducción}

Los devastadores efectos de los sismos que continuamente se producen mantienen despierto el interés general por comprender sus causas y mecanismos y el interés en anticiparlos y reducir sus efectos sobre vidas y bienes. Como consecuencia de ello se generan materiales de divulgación y de apoyo a la enseñanza del tema, de los que existe una gran oferta. No son habituales, sin embargo, los trabajos de análisis de los aciertos y falencias de los modelos analógicos que habitualmente se incluyen en esos materiales, a pesar de que es tan importante saber qué hace bien un modelo o una demostración como saber qué es lo que no hace o hace mal. Presentar estos factores a quienes aprenden permitiría evitar malentendidos, las apreciaciones falsas o, incluso, que llegue a tomarse el modelo por una versión de tamaño reducido de una realidad que es siempre muchísimo más compleja.

\begin{tabular}{c|c|c|c|c|c|}
\hline (C) Terrae Didat. & Campinas, SP & v.16 & $1-11$ & $\mathrm{e} 020029$ & 2020 \\
\hline
\end{tabular}


Esta contribución se propone, por lo tanto, promover la reflexión acerca de los alcances y limitaciones de cada modelo, de forma que los mismos puedan ser advertidos y transmitidos a los alumnos. Es importante resaltar que los procesos y fenómenos que se vinculan a la actividad sísmica deben ser abordados desde una perspectiva interdisciplinaria, ya que involucran tanto conceptos de Ciencias de la Tierra como de Física, Matemáticas y Ciencias Geográficas y Sociales.

A continuación se presenta una lista de los procesos y fenómenos vinculados a los terremotos y susceptibles de ser modelizados que serán considerados en la presentación:

Acumulación y liberación de energía

- Mecanismos de acumulación de energía

- Mecanismos de liberación de energía

- Movimiento del suelo

- Efectos de la presencia y presión de agua en el comportamiento de los materiales

Transmisión de la energía liberada

- Movimiento de las partículas

- Generación de ondas sísmicas

- Transmisión de ondas sísmicas

- Diferenciación de los distintos tipos de ondas

Los modelos vinculados a los efectos de las ondas sísmicas en las construcciones civiles y a su mitigación, así como su incidencia en la estabilidad del terreno (solifluxión, avalanchas, etc.) serán considerados en sendas contribuciones que ya se encuentran en preparación avanzada.

\section{Descripción de las actividades}

La descripción se desarrolla en formato de ficha que incluye: a) una introducción al área conceptual; b) una descripción de la actividad, sus materiales y métodos; c) su vinculación con la situación real y d) aquéllos puntos en que el modelo es adecuado y los en los que no lo es. En algunos casos se han incluido comentarios que amplían el tratamiento del tema o actividad en cuestión.

\section{Acumulación y liberación de energía}

Para comprender los mecanismos de liberación de energía por desplazamiento sobre una superficie de fractura (nueva o preexistente) es necesario recurrir a los conceptos de fuerza y esfuerzo. Estos conceptos están estrechamente vinculados a los procesos de fracturación y desplazamiento de las masas de la roca. Sus relaciones pueden enfatizarse si se analizan las diferentes situaciones que tienen origen cuando se ejerce una fuerza sobre un sólido tomando en consideración las posibilidades de que éste pueda o no desplazarse como resultado de la aplicación de esa fuerza.

a. El sólido puede desplazarse. En ese caso la aplicación de la fuerza genera el movimiento y el sólido continúa su desplazamiento hasta tanto otra fuerza no se oponga al mismo (obstáculo) o se desarrolle un proceso que facilite la disipación de la energía involucrada (rozamiento, generación de calor).

b. El sólido no puede desplazarse. En este caso surgen dos alternativas principales. La primera opción es que el sólido pueda deformarse. En este caso la fuerza se disipa en forma de trabajo, que es realizado para desplazar las partículas unas con respecto a otras y producir el cambio de forma, aunque no haya un desplazamiento apreciable del conjunto (como ocurre cuando se presiona una bolita de masilla colocada sobre una mesa). En la segunda, si el sólido es compresible, reacciona cambiando su volumen y "almacenando" la energía resultante (caso de un resorte), pero si es incompresible (y dado que no puede desplazarse) la aplicación de la fuerza genera en el mismo un estado de tensión (caso de un bloque no deformable). En este último caso todas las partículas constitutivas del bloque alteran sus relaciones y se produce una distribución tridimensional de la fuerza en el interior del cuerpo, generándose esfuerzos, de modo similar (ipero no exactamente igual!) a como se generan las presiones al comprimir un gas. Las propiedades mecánicas del material definirán el momento en que éste ya no puede resistir las tensiones internas y se rompe. Al no haber habido una disipación previa de la energía (como podría ser la consumida en forma de trabajo al generar cambios en la forma del cuerpo), la energía acumulada en la red de partículas del sólido es liberada en forma brusca y se produce la rotura a lo largo de una superficie, denominada fractura. Toda la energía sobrante de la fracturación será utilizada en el desplazamiento de los fragmentos del bloque (la fractura se transforma en falla) y en la generación de calor. Un razonamiento similar cabe cuando existe una fractura previa y el material cede frente al esfuerzo a lo largo y a lo ancho de esa superficie preexistente al superarse la resisitencia opuesta por la fricción. Este el caso más habitual en los sismos. 
Si la fuerza que ha producido el fenómeno es del tipo no renovable, una vez generada la fractura y producido el desplazamiento, el sistema vuelve a un estado de reposo. Si la fuerza es de tipo renovable (como es el caso de las fuerzas generadas por los desplazamientos de las placas litosféricas) el sistema volverá a tensionarse y deformarse una y otra vez. Las relaciones entre las fuerzas aplicadas, las direcciones de movimiento relativo de los bloques y los denominados "mecanismos focales" (que permiten reconstruir esas direcciones de movimiento relativo a partir de la información sísmica) han sido desarrollados en Sellés-Martínez \& Bonán (2000) y en Sellés-Martínez (2005).

\section{Actividad 1: Un zapateado planetario}

Descripción: Se trata de una actividad de presentación del tema. Es la simplicidad de la experiencia la que la hace especialmente adecuada para utilizarla en el inicio de la presentación de estos temas en clase y en contextos de divulgación.

Materiales y metodología: Se requiere un juguete a cuerda que reproduce la forma del planeta con la adición de un par de piernitas y unos grandes pies que, al liberarse la energía acumulada en la cuerda, hacen que el juguete zapatee (Fig. 1).

La experiencia es simple. Se da cuerda al juguete, se lo apoya sobre una superficie plana y se deja que ejecute su zapateado. A continuación se solicita a los asistentes que describan el proceso de conservación de la energía involucrado. Surgirá, seguramente, la propuesta de la acumulación de energía en el resorte y su posterior liberación en el zapateo.

Análisis de la experiencia y vinculación al proceso real: El juguete parecería no tener más relación con el tema que su forma "planetaria", sin embargo más allá de esa simple apariencia formal, el mecanismo del muelle espiral en el interior del juguete es un mecanismo de acumulación y liberación de energía. Es importante comparar si la forma de liberación de la energía es similar en el modelo y la realidad. La liberación de la energía acumulada en una zona sísmica es gradual cuando se la considera a la escala de los tiempos y procesos geológicos y tiene lugar a través de una muy grande cantidad de sismos de magnitudes muy diferentes; pero es casi instantánea si se la considera a escala de cada sismo y del tiempo en la vida cotidiana. El análisis detallado de cada sismo muestra, a su vez, que la ruptura y desplazamiento sobre el plano de falla no son simultáneos en toda su superficie, pero el evento

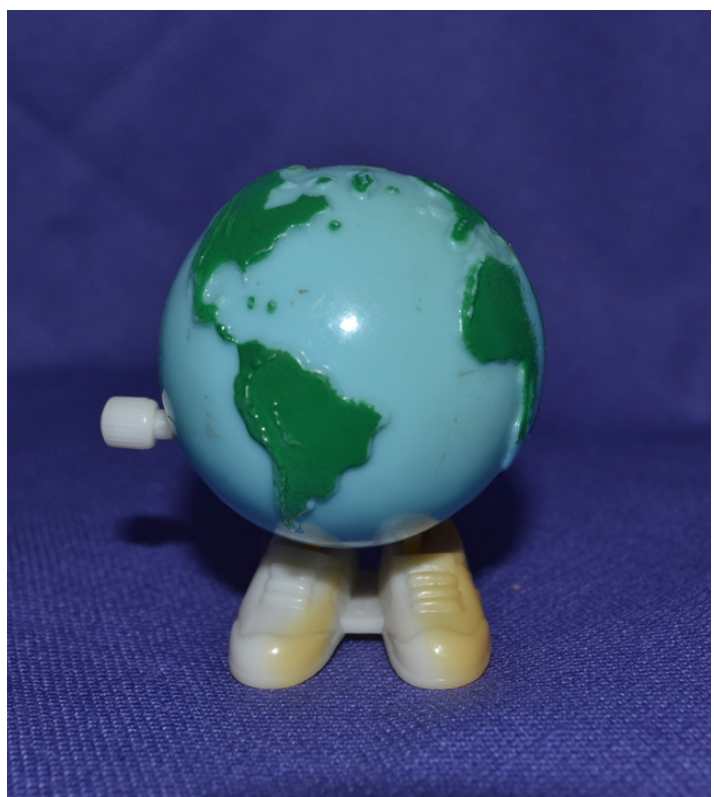

Figura 1. El planeta zapateador vibra cuando se libera la energía del resorte que lleva en su interior

total sólo dura unos pocos segundos o, a lo sumo, escasos minutos.

Aspectos que son reproducidos aceptablemente: los procesos de acumulación y liberación de energía (que resulta en un movimiento) son procesos equivalentes en el modelo y la realidad. Si se trabaja con alumnos más avanzados puede señalarse que, en el ámbito de la realidad, se trata de un proceso de liberación de la energía acumulada por la aplicación de una fuerza sobre un material que se deforma elásticamente hasta que se alcanza su resistencia límite y el material se rompe o cede sobre las superficies de debilidad pre-existentes en el mismo, Coincide en la realidad y en el modelo la necesidad de, una vez consumida la disponible, volver a acumular energía en el sistema para que el sistema pueda moverse nuevamente. Entre los puntos de interés a destacar puede señalarse que, al igual que en los terremotos reales de una magnitud importante, es toda la Tierra la que vibra como consecuencia de un sismo.

Aspectos en los que el modelo no es fidedigno: A diferencia del juguete, la liberación de la energía de la Tierra no está controlada por un mecanismo que la transforma en un movimiento de vaivén en las "piernas" del juguete, sino que irradia desde el lugar en que se ha producido el sismo, en todas direcciones, como un conjunto de ondas que, a su vez, activan las partículas a través de las cuales se propagan. Puede agregarse que casi toda la energía 
acumulada por el resorte en el muñeco es transformada en movimiento, sin embargo (y afortunadamente) la mayor parte de la energía liberada en un sismo no se transforma en ondas sino que es consumida como trabajo para desplazar las masas involucradas en la fractura y, sobre todo, es disipada como calor (aproximadamente el 90\%).

Comentarios: Puede discutirse con los alumnos que posibilidades de controlar la velocidad de liberación de la energía por parte del resorte existen y cual sería su correlato con la realidad geológica. Las respuestas más sencillas a estas cuestiones podría ser el control que sobre los resortes espirales de los relojes (cronógrafos antiguos, relojes cu-cu) se establecen con mecanismos compensadores (como los volantes y las pesas) que hacen más lenta y pareja la liberación de la energía, ya que de otro modo las manecillas del reloj correrían muy rápido al principio, cuando hay mucha energía acumulada, pero muy lentamente hacia el final, cuando aquélla es ya poca. En el ámbito geológico puede señalarse que la existencia de jaboncillos de falla sobre la superficie de las fracturas, al disminuir el valor del coeficiente de rozamiento entre los bloques, facilita su desplazamiento cuando aún son bajos los esfuerzos y tiende a producir un desplazamiento cuasi continuo, asociado a muchísimos sismos de muy baja magnitud, en lugar de pocos sismos muy fuertes y más destructivos.

\section{Actividad 2: Deformación "a medida"}

Descripción: Con un muelle o resorte lineal puede representarse el proceso de acumulación y liberación de la energía simplemente comprimiendo el mismo y soltándolo repentinamente. Una experiencia más detallada es la constatación de la Ley de Hook, que establece que la elongación o estiramiento del muelle o resorte es proporcional a la fuerza que se ejerce sobre el mismo y dicha proporcionalidad está vinculada a las características del resorte.

Materiales y metodología: Uno o varios muelles o balanzas del tipo "dinamómetro" (Fig. 2), algunas pesas y una regla para medir el estiramiento del resorte son necesarios para realizar la experiencia. Si el resorte es pequeño basta con adicionar al mismo un pequeño recipiente e ir colocando en él pesos conocidos (pueden ser monedas iguales) y medir la longitud a cada paso. Puede utilizarse también una balanza de uso común si se le cuelga una bolsa o recipiente en el que puedan ir colocán- dose libros $\mathrm{u}$ objetos pesados y se va midiendo la distancia del cero al peso alcanzado en cada oportunidad. Por diferencia en las lecturas de la balanza se calcula cuánto peso se ha agregado cada vez. Si esos datos se representan en un gráfico cartesiano puede verse que los puntos se alinean en una recta cuya pendiente es función de las características de cada resorte. (denominada constante $K$ del resorte). El concepto se ve fuertemente reforzado si se dispone de dos o tres resortes diferentes y se repite la experiencia con cada uno de ellos. La pendiente de la recta obtenida en el gráfico es la relación entre la fuerza aplicada y la deformación causada y representa tanto la constante del resorte usado como modelo, como el módulo de elasticidad del sólido que se está modelando.

Análisis de la experiencia y vinculación al proceso real: Valen aquí muchos de los comentarios realizados para el caso de la actividad 1, con la diferencia que al trabajar con el resorte lineal pueden establecerse relaciones cuantitativas entre la fuerza y la deformación que no era posible realizar al utilizar el resorte o cuerda en espiral. Se verifica la expresión de la Ley de Hook que establece que la deformación (D) es directamente proporcional a la fuerza aplicada $(\mathrm{F})$ multiplicada por la constante $(\mathrm{K})$ que caracteriza al muelle $(\mathrm{D}=\mathrm{K} \mathrm{F})$

Aspectos que son reproducidos aceptablemente: El proceso de acumulación y liberación de energía es modelado adecuadamente y puede verse la relación directa entre la cantidad de energía acumulada y la magnitud de la deformación producida en el muelle, pero que esta última está, sin embargo, controlada por las características del muelle (equivalente a las propiedades mecánicas de los mate-

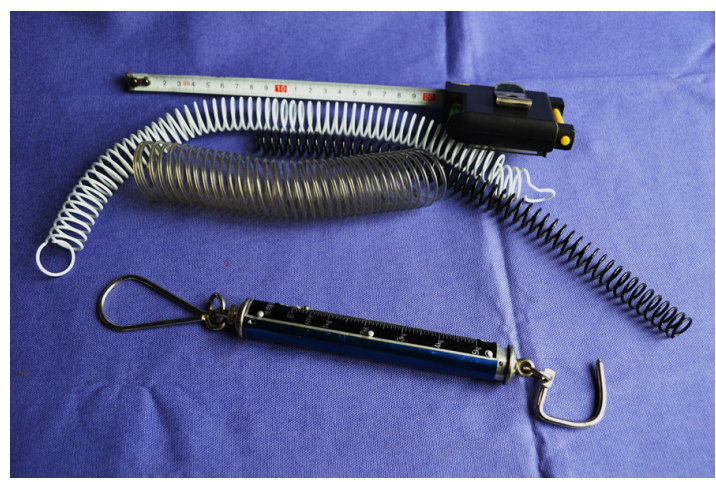

Figura 2. Balanza a resorte que permite medir la proporcionalidad entre el peso y la deformación del muelle. Puede ser reemplazada por resortes plásticos y un metro para medir la elongación que tiene lugar cuando se adiciona una carga 
riales geológicos). Otro punto de relación positiva entre los muelles y la realidad geológica es el hecho que la deformación puede producirse en contextos compresivos (comprimir el resorte) o tractivos (estirar el resorte).

Aspectos en los que el modelo no es fidedigno: La deformación del muelle se da siempre en una única dirección (deformación longitudinal) mientras que en los procesos reales la situación se da más comúnmente en contextos tridimensionales, aún cuando por razones de simetría o de magnitudes relativas de las fuerzas actuantes, los esquemas analíticos puedan ser reducidos a sistemas de sólo dos dimensiones.

\section{Actividad 3: Jalando ladrillos}

Descripción: Esta actividad se encuentra descripta en http:/www.earthlearningidea.com/PDF/49 _Spanish.pdf y su objetivo es verificar que aún bajo condiciones aparentemente iguales resulta muy difícil predecir el momento en el que se producirá el desplazamiento sobre la superficie de fractura. Es importante tener en cuenta que se está modelando la acumulación de energía en dos bloques unidos por un plano de falla preexistente. La energía suministrada es acumulada hasta que la misma supera el valor necesario para vencer la inercia del cuerpo y ponerlo en movimiento. Dado el rozamiento entre las superficies, el movimiento se extinguirá como consecuencia de la disipación de la energía y el bloque volverá a una posición de reposo pero en un punto diferente al inicial.

Materiales y metodología: El equipamiento consiste en un cordel y un ladrillo apoyado sobre otro ladrillo (si se desea que ambas superficies sean iguales) o sobre una superficie plana cualquiera. Si se varía el peso del bloque (adicionando otro ladrillo sobre él) o se varía la fuerza de rozamiento entre el bloque y la superficie de deslizamiento (cubriéndola con una película de cera o interponiendo una hoja de papel) se puede verificar que la fuerza necesaria para poner el bloque en movimiento es diferente en cada caso.

Análisis de la experiencia y vinculación al proceso real: La experiencia vincula claramente la complejidad del proceso de predicción del momento en que el bloque se desplazará (momento en que se producirá el sismo). Esto es así aún cuando las condiciones de contorno sean conocidas y el proceso sea, en términos estadísticos, predecible.

Aspectos que son reproducidos aceptablemente: El proceso de acumulación y liberación de energía es reproducido adecuadamente y se evidencia también que existe una relación entre las características de la superficie de contacto entre los bloques y la fuerza necesaria para producir el desplazamiento. Si la experiencia se realiza utilizando dos ladrillos nos encontramos frente a un caso geológico en el que los bloques a ambos lados del plano de falla son de una misma unidad litológica o muy similares (por ejemplo granito sobre granito) pero si son diferentes podemos establecer la correlación con el caso en que los bloques son diferentes, por ejemplo calizas sobre granitos. Estos contactos entre materiales diferentes se asocian directamente a la magnitud del desplazamiento anterior sobre esa superficie de falla y a las dimensiones de las unidades geológicas (cuerpos de roca) involucradas.

Aspectos en los que el modelo no es fidedigno: En la mayoría de los casos reales la fuerza aplicada es permanente en el tiempo (ya que el movimiento de las placas se mantiene en la escala de tiempo involucrada) y lo que se disipa es la energía acumulada elásticamente pero sin anularse la fuerza. En el modelo esto es difícil de lograr ya que una vez que el ladrillo se pone en movimiento la cuerda pierde tensión y, al aflojarse, desaparece la fuerza aplicada. Un problema adicional lo constituye el hecho que dado que el coeficiente de rozamiento estático de los materiales es generalmente mayor que el coeficiente de rozamiento dinámico, por lo cual, una vez puesto en movimiento el bloque será difícil detenerlo rápidamente si la fuerza aplicada permanece constante (como sería el caso en que se trabaje acumulando peso en un recipiente) y el modelo, en ese punto, se asemejaría más a una deformación plástica que a una frágil.

Comentarios: 1. Una variación interesante y simple para tener una idea aproximada de cuánta fuerza ha sido necesario ejercer para lograr el desplazamiento consiste en vincular, mediante un cordel, el bloque y un recipiente en el que se adicionará peso (por ejemplo naranjas o patatas pequeñas) paso a paso hasta que el bloque pierda estabilidad. 2. La actividad ofrece también la alternativa de controlar la fuerza que se ejerce mediante un dinamómetro (como en el caso anterior puede servir una balanza de las que se usan para pesar pequeños paquetes). El artículo de Pantosti (2010) complementa adecuadamente este ítem y el próximo, exponiendo las complejas relaciones que se generan entre el conocimiento del fenómeno sísmico, su predictibilidad y las implicaciones sociales de dar o no una alarma sísmica. 


\section{Actividad 4: El valor del rozamiento reviste gravedad}

Descripción: Esta actividad es una variación de la anterior que pone en juego las relaciones entre las fuerzas aplicadas, las características de la superficie de contacto entre los bloques y la posibilidad de que se produzca el desplazamiento. En este caso se pone en evidencia, además, la influencia de la orientación de la superficie de discontinuidad con respecto a la dirección de aplicación de la fuerza. Esto se debe a que el coeficiente de rozamiento está vinculado directamente al valor de la componente de la fuerza aplicada que actúa en dirección perpendicular a la superficie en estudio.

Materiales y metodología: Son necesarios un recipiente de forma prismática o caja pequeña, una tabla, una serie de bloques de madera o elementos similares que permitan aumentar o disminuir la inclinación de la tabla y un conjunto de pequeños pesos para incrementar la carga en el recipiente (Fig. 3). Se exploran dos alternativas: a) para un mismo valor de carga en el recipiente se aumenta progresivamente el ángulo de inclinación y b) para un mismo ángulo de inclinación se aumenta progresivamente la carga. En el primero, el umbral se alcanza porque al aumentar el ángulo de inclinación disminuye la componente de la fuerza de gravedad perpendicular al plano y por lo tanto se reduce también la fuerza de rozamiento estático. En el segundo la componente paralela al plano aumenta porque aumenta la fuerza total aplicada. Lo que ocurre en los casos reales se vincula más al segundo caso ya que aumenta la fuerza acumulada; pero también puede ocurrir que el progreso de la deformación del sistema rocoso produzca la rotación de las estructuras preexistentes y, al establecerse nuevas relaciones entre la orientación de las superficies y la de las fuerzas aplicadas, se induce el desplazamiento sobre superficies que eran inactivas.

\section{Análisis de la experiencia y vinculación al proceso real: Valen los señalados para la actividad 3.}

Aspectos que son reproducidos aceptablemente: A los señalados para la actividad 3 , puede sumarse que pueden encontrase ejemplos de la rotación de los planos de estratificación en el desarrollo del proceso de plegamiento (deformación progresiva) y, a escala litosférica, en la flexión de la placa que se introduce en la zona de subducción que, al pasar de una posición horizontal a una posición inclinada (que

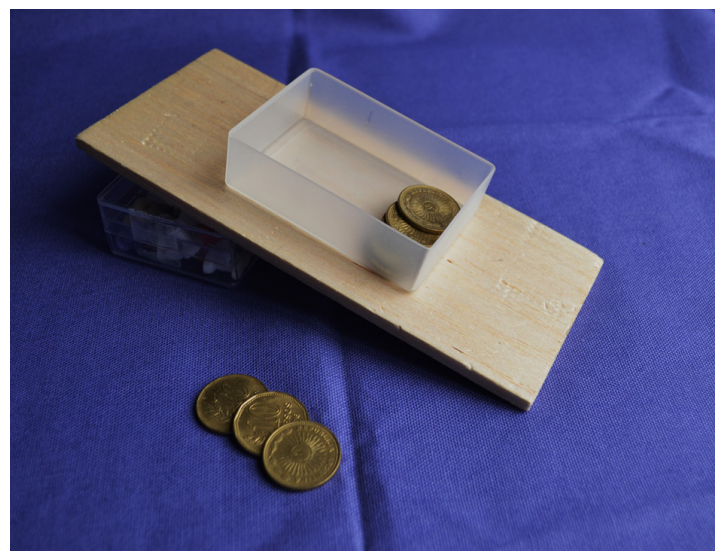

Fig. 3. Un recipiente hueco permite regular su carga y ver cómo influye el ángulo de inclinación en la composición de fuerzas, facilitando o dificultando el inicio del movimiento. Esto ocurriría cuando una fractura geológica gira con respecto a la dirección de las fuerzas que actúan regionalmente

llega a ser en algunos casos excepcionales empinada o casi vertical). Las fracturas y discontinuidades mecánicas (planos de estratificación y de clivaje) preexistentes son así reorientadas con respecto al campo regional de esfuerzos, que permanece estable en su orientación.

Aspectos en los que el modelo no es fidedigno: La fuerza que actúa en la experiencia es el peso, asociado a la gravedad mientras que en la realidad, aún cuando la fuerza de gravedad se encuentre presente, en la mayoría de los casos (ambientes compresivos en los que se activan fallas inversas o desplazamientos de rumbo), la fuerza que condiciona la puesta en movimiento sobre la superficie de falla es de origen tectónico, salvo que se trate de un ambiente extensional. En este caso la fuerza de gravedad genera la actividad sísmica por deslizamiento sobre fallas de tipo directo.

\section{Transmisión de energía en forma de ondas}

Debe señalarse que el concepto geométrico de onda, línea ondulante, atenta contra el concepto de onda como forma de transmisión de la energía. En primer lugar porque la primera se desarrolla en forma bidimensional mientras que las ondas sísmicas son tridimensionales. La representación gráfica (cartesiana) de la posición espacial de las partículas (eje vertical) en función del tiempo (eje horizontal) durante un movimiento oscilatorio armónico puede contrastar por lo tanto con el desplazamiento real de las partículas si nos referimos a la transmisión de una onda de presión. Así, las espiras de un resorte (que se acercan y se separan 
siempre en la dirección del eje del mismo) no se aprecian como la ondulación o serpenteo que evoca una onda, sino como el pasaje longitudinal de un impulso de contracción y extensión. Estas diferencias (dirección real del movimiento de la partícula vs. su representación cartesiana) son una causa de confusión en la comprensión. Es justamente por esto que, a nuestro criterio, es importante no utilizar el resorte como modelo para simular ondas $\mathrm{S}$ y hacerlo con una cuerda que, como no es elástica, no puede usarse para simular ondas $\mathrm{P} y$, así evitar el uso del mismo elemento para simular cosas diferentes, lo que puede dificultar la comprensión.

Un buen ejemplo para abordad el problema es tomar como fuente de las ondas una explosión subterránea, de dimensión puntual a escala de la Tierra. Esta explosión se propagará en todas direcciones como una onda $\mathrm{P}$ y, como no ha habido desplazamiento lateral de materia en el punto de explosión sino sólo compresión radial, la explosión no generará ondas S (esto es sólo teóricamente cierto, la realidad es un poco más compleja, pero el ejemplo es válido haciendo esta salvedad). Por otra parte, si un fleje metálico fijo por un extremo a un cuerpo sólido es flexionado, al liberarlo comenzará a vibrar, retenido por el punto de fijación. Las ondas que se transmiten del fleje al sólido en el que está fijado son ondas S, y no necesariamente deberían generarse ondas $\mathrm{P}$, ya que no ha existido ni compresión ni expansión en el cambio de forma del fleje (pero nuevamente debe hacerse la salvedad de que en la realidad las ondas $\mathrm{S}$ pueden generar ondas $\mathrm{P}$ en los sólidos a los que llegan). Sin embargo, la vibración del fleje en el aire sí generará ondas compresivas en éste, pues comprime y rarifica cíclicamente la masa que está por delante y detrás de sí.

\section{Actividad 5: Escrito en el agua}

Descripción: Los círculos concéntricos que se amplían a partir del punto de la superficie del agua en el que ha caído una piedra son el ejemplo más frecuente cuando se quiere describir una onda. Materiales y metodología: Se trata simplemente de arrojar una pequeña piedra en un recipiente adecuado (lo más amplio posible) lleno de agua y observar la generación y propagación de las perturbaciones en la superficie del líquido.

Análisis de la experiencia y vinculación al proceso real: el modelo es de utilidad para ejemplificar algunos conceptos como: a) la formación de frentes de propagación circulares, que se correlacionarían con frentes esféricos en el caso de un sismo real, b) la presencia de zonas elevadas y deprimidas en la superficie del agua, que se correlacionan con las zonas de compresión y expansión en el caso de las ondas de cuerpo, c) se pueden ver también claramente los fenómenos de reflexión en los bordes del recipiente y de interferencia entre las ondas primarias, las de las primeras reflexiones y las de los subsiguientes, del mismo modo que este fenómeno ocurre, en tres dimensiones, en el interior de la Tierra.

Aspectos que son reproducidos aceptablemente: Están incluidos en el párrafo anterior.

Aspectos en los que el modelo no es fidedigno: El tipo de onda generado en la interfase entre el agua y la atmósfera no es equiparable a las ondas $\mathrm{P}$ y S, que son las que se utilizan para analizar los sismos, sino con las ondas de superficie (como las Raleigh y las Love) que, si bien son las responsables del efecto destructivo del sismo, habitualmente no son incluidas en las explicaciones. La diferencia entre las amplitudes relativas de las ondas $\mathrm{P}$, S y superficiales puede ser claramente advertida observando un sismograma típico.

Comentario: El análisis de la secuencia de la película Parque Jurásico en la que las pisadas del dinosaurio se perciben dentro del vehículo como ondas en la superficie del agua contenida en un vaso, ilustra claramente como una fuerza (relacionada al peso del dinosaurio) impacta contra el suelo, al que transmite una cierta cantidad de energía. Ésta se propaga en todas direcciones como una onda. Esa onda alcanza a todos los elementos apoyados en el suelo y los hace vibrar. Las vibraciones de la masa de agua (ondas P), se convierten en ondas superficiales en la interfase entre el agua y el aire. No ha sido necesario, en este caso, que cayera una piedra en la superficie del agua para producir los anillos concéntricos.

\section{Actividad 6: Ondas, muelles y cuerdas que poco tienen que ver con un puerto}

Descripción: El título de la actividad se propone, si se está trabajando con estudiantes no muy avanzados o en un ámbito de divulgación, someter a consideración la polisemia de muchos de los términos utilizados como una forma de introducir el tema y asegurarse que todo el mundo entiende claramente de qué se está hablando. Puede preguntarse a los alumnos que diferencias hay entre el uso de la palabra onda en "el cabello le caía en ondas sobre los hombros", "La transmisión se efectuó en un equipo de onda corta" y "las ondas 
sonoras se transmiten más rápido en el metal que en la madera". Con respecto a "muelle" pueden utilizarse las expresiones "muelle de pescadores", "una vida muelle" o "se estiraba y contraía como un muelle" y, finalmente puede decirse que es bueno que alguien sea "una persona cuerda" pero malo que termine sus días "colgado de una cuerda". La parte experimental se propone demostrar como un breve impulso de compresión o alivio que se imprime a un muelle o resorte en la dirección de su longitud permite simular la propagación de las ondas P (o de presión) mientras el movimiento sinusoidal que puede imprimirse a una cuerda simula el desplazamiento relativo de las partículas al paso de una onda S (o de cizalla).

Materiales y metodología: Se utilizará un resorte blando para simular las ondas P y una cuerda de un par de metros de longitud para las S. La simulación es simple y la actividad se encuentra descripta en http://www.earthlearningidea.com/PDF/76_Slinkies.pdf. La experiencia puede realizarse consiste en desplazar los extremos del resorte hacia fuera o hacia adentro en forma repentina y observar como el impulso se transmite a lo largo del muelle. En el caso de cuerda, la misma es sacudida lateralmente para imprimirle un movimiento serpenteante. Se observa como la perturbación se transmite a lo largo de la cuerda, pero el movimiento de cualquier punto de ésta está contenido en una línea que es perpendicular a la dirección de avance de la perturbación (coincidente con la orientación de la cuerda).

Análisis de la experiencia y vinculación al proceso real: La aplicación más importante de los muelles es la posibilidad de visualizar el modo como un impulso determinado se transmite a lo largo de un cuerpo, lo deforma transitoriamente desplazando una porción del mismo y como, luego del paso de la perturbación, aquél recupera su forma original cuando la porción desplazada recupera su posición. Así como el muelle no resulta el modelo más adecuado para reproducir las ondas de cizalla, la soga es completamente inútil para representar las ondas longitudinales.

Aspectos que son reproducidos aceptablemente: Como se señaló algo más arriba, el muelle es un modelo muy adecuado para representar las ondas $\mathrm{P}$, ondas de presión que se propagan como un movimiento hacia atrás y adelante de las partículas, en la dirección de la propagación de la onda, o más exactamente, como un proceso de compresión y expansión del medio, en frentes esféricos consecutivos, si se piensa el proceso en tres dimensiones. A este respecto puede completarse la actividad fijando la atención en un grupo determinado de espiras (que pueden marcarse con pintura de color) y viendo como las mismas cambian cíclicamente la distancia entre ellas al paso de la perturbación. Puede filmarse un video y analizarlo para cuantificar las distancias mínima, media y máxima de separación entre las espiras.

Aspectos en los que el modelo no es fidedigno: En el caso del muelle, éste no permite visualizar adecuadamente el comportamiento de las partículas (moléculas) que son alcanzadas por la vibración y cuyo movimiento no constituiría realmente un "desplazamiento" visible. Este inconveniente puede solucionarse, al menos parcialmente, complementando esta actividad con la que se describirá a continuación. Con respecto a la cuerda, es importante destacar que no debe hacerse una transferencia inmediata de la forma serpenteante de la cuerda con la representación cartesiana de posición en función del tiempo para cada punto de la cuerda, ya que, como se señaló antes, esto es sólo una coincidencia geométrica y el gráfico es visualmente igual cuando se representa en coordenadas similares el apartamiento de una partícula alcanzada por una onda $\mathrm{P}$ desde su posición de reposo. Por otra parte, es fácil ver que en un sistema real, el hecho de comenzar a vibrar no exige un acercamiento de dos puntos cualesquiera del sistema pero en el caso de la cuerda es necesario que los puntos extremos de la misma se acerquen para que la misma pueda serpentear en forma apreciable, ya que no es igual la longitud de una línea recta que la de una sinusoide que unen los puntos que corresponden a los extremos de ambas. En el caso real la distancia entre moléculas o partículas que transmiten el movimiento aumenta y disminuye al paso de la onda, pero esto no es apreciable megascópicamente.

Comentario: Puede verse también que, en la experiencia, la frecuencia de la vibración influye sobre la amplitud de la ondulación e incluso sobre el alcance del impulso.

\section{Actividad 7: Navidad es tiempo de buenas ondas}

Descripción: Este modelo fue propuesto por el autor como una simplificación de otros, más precisos pero más costosos, que se construyen con esferas de acero (http://www.earthlearningidea.

com/PDF/121_Merry_waves.pdf). El objetivo de este modelo es visualizar que la transmisión del

\begin{tabular}{c|c|c|c|c|c}
\hline C Terrae Didat. & Campinas, SP & v.16 & $1-11$ & e20029 & 2020 \\
\hline
\end{tabular}


impulso en la propagación de la onda no implica el desplazamiento físico apreciable de los materiales involucrados

Materiales y metodología: Unas seis u ocho bolitas de las que se utilizan para el árbol de Navidad (de igual tamaño) son colgadas de un soporte horizontal (una percha) mediante un hilo que pasa a través del aro de todas ellas y sobre el travesaño horizontal de la percha (Fig. 4). Se ajustan las espiras del hilo con una regla de modo que las bolitas queden alineadas y en contacto unas con otras. Se aparta suavemente la bolita ubicada en uno de los extremos y se la deja caer. Como consecuencia del golpe de ésta con la segunda bolita en la fila, la última saldrá proyectada hacia delante, sin que las intermedias se hayan desplazado.

Análisis de la experiencia y vinculación al proceso real: La experiencia muestra como una partícula alcanzada por un impulso lo trasmite a otra con la que está en contacto.

Aspectos que son reproducidos aceptablemente: El concepto de transmisión del impulso o energía vibratoria de una molécula a la siguiente, y de ésta a la que le sigue y así sucesivamente, es reproducido adecuadamente.

Aspectos en los que el modelo no es fidedigno: La transmisión del impulso se realiza sólo en la dirección de la fila y, aún cuando se montara una red tridimensional (o simplemente plana) sería sumamente difícil reproducir la tridimensionalidad de las ondas reales, cuyos frentes se propagan en todas direcciones y en patrones esféricos, independientemente del tipo de onda.

Comentarios: 1. Puede complementarse esta demostración intentando simular con el mismo montaje, la propagación de una onda S. Al desplazar la primera bolita en dirección perpendicular a la fila y dejarla caer, la bolita oscilará un rato alrededor de su posición de reposo, como si fuera un péndulo, pero apenas moverá (por efecto del rozamiento eventual en el punto de contacto entre ambas) a la bolita siguiente. Al no existir vínculo entre ambas bolitas el desplazamiento lateral, perpendicular a la dirección de la fila, no se transmite. 2. La transmisión del impulso a lo largo de una fila de partículas puede hacerse también con una fila de monedas colocadas sobre una superficie plana. Cuando se golpea lateralmente (puede hacerse deslizando una regla sobre la superficie plana) la primera de ellas la última sale disparada, apartándose de la punta de la fila tanto como fuerte haya sido el golpe en la primera. No

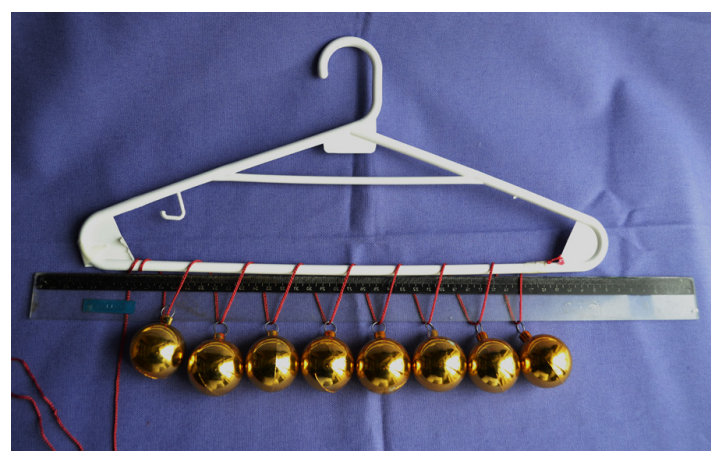

Figura 4. Las bolitas de Navidad, colgadas en forma alineada de una percha, permiten simular la transmisión de un impulso a través de la materia sólida

ha habido desplazamiento perceptible pero ha habido una eficiente transmisión del impulso.

\section{Actividad 8: Las ondas P atacan por la espalda}

Descripción: El uso de una fila de estudiantes para representar las características de las ondas $\mathrm{P}$ y las ondas $S$ puede ser una forma amena y de interés didáctico, para dar comienzo o cierre a una clase sobre el tema.

Materiales y metodología: La experiencia (descripta en http://www.earthlearningidea.com/

PDF/77_Human_molecules.pdf), es simple y efectiva. Consiste en colocar un cierto número de alumnos en fila (cinco a siete) y a una corta distancia uno de otro. Los alumnos de la fila sólo ven la espalda del compañero que tienen delante y no saben qué pasa detrás de ellos. El docente, en forma imprevista, empuja hacia delante al último de la fila quién, antes de enderezarse instintivamente, empujará inevitablemente al compañero que tenía adelante y, así sucesivamente hasta llegar al primero, que se tambaleará antes de recuperar su posición erguida en la fila. En los momentos inicial y final de la experiencia los alumnos se encuentran en la misma posición, pero ha habido un momento intermedio, durante el paso de la onda que los ha "deformado" y que ha hecho que la energía recibida desde el alumno que tenían detrás fuera transmitida al que tenían delante, en que sus torsos se han desplazado. Si en lugar de empujar al último de la fila hacia delante se lo desplaza ligeramente hacia el costado tomándolo de un brazo, el resto de los miembros de la fila no percibe que esto ha ocurrido. No se ha generado una "onda S" porque no existe un vínculo adecuado entre las partículas. $\mathrm{Si}$ los alumnos en la fila giran $90^{\circ}$ y se colocan lado a lado, enlazando sus brazos, se habrá generado un

\begin{tabular}{c|c|c|c|c|c}
\hline (C) Terrae Didat. & Campinas, SP & v.16 & $1-11$ & $\mathrm{e} 020029$ & 2020 \\
\hline
\end{tabular}


vínculo entre partículas y, ahora sí, un pequeño tirón o empujón lateral al último alumno hará ondular la fila hacia derecha e izquierda, mientras la onda se propaga de una punta a la otra de la hilera de estudiantes.

Análisis de la experiencia y vinculación al proceso real: En general valen también en este caso los conceptos ya expresados para las actividades anteriores. Otros se incluyen en el punto siguiente.

Aspectos que son reproducidos aceptablemente: Este modelo permite poner claramente en evidencia que, a diferencia de las ondas $\mathrm{P}$, en el caso de las ondas $\mathrm{S}$ es necesario que existan fuerzas que vinculen a las partículas entre sí para que el impulso se transmita. Puede señalarse la relación entre lo ocurrido en un caso con el comportamiento de los sólidos (que transmiten tanto las ondas $\mathrm{P}$ como las S) y en el otro con el de los líquidos, que solo transmiten las ondas P, pues al carecer de resistencia a la cizalla no pueden transmitir las $S$ ya que, simplemente, se deforman de modo plástico (es decir sin posibilidad de volver a recuperar su forma inicial al recibir el impulso).

Aspectos en los que el modelo no es fidedigno: En términos generales el modelo es bastante adecuado a lo que se quiere modelar y, más allá de las limitaciones lógicas de escala y las diferencias entre los lazos físicos que se establecen entre los individuos (que enlazan "materialmente" sus brazos) y las fuerzas de atracción molecular (inmateriales) que vinculan las partículas en los materiales sólidos y líquidos, no existen problemas en la simulación.

\section{Actividad 9: Un picnic estropeado}

Descripción: Cuando se hace referencia a las ondas sísmicas suele no hacerse demasiada referencia a las ondas de superficie (Love y Raleigh, por ejemplo) aunque se mencione que son las más destructivas. Su modelado no resulta sencillo, ya que los desplazamientos de las partículas al paso de estas ondas son complejos y a diferencia de los casos de las ondas P y S, no son ni paralelos ni perpendiculares a la dirección de transmisión del impulso.

Materiales y metodología: Esta actividad (icon el propósito de reducir su costo!), puede llevarse a cabo sólo con auxilio de la imaginación. La propuesta consiste en imaginar que se agita un mantel sobre el que se han dispuesto todos los elementos de la merienda que se ha preparado para un día de campo. ¿Qué ocurrirá si un perro muerde uno de los extremos del mantel y comienza a sacudir desordenadamente el mismo hacia arriba y hacia abajo repetidamente desde uno de sus lados? Las ondulaciones y vaivenes de la tela provocarán la caída de todo lo que esté apoyado sobre ella. Puede preguntarse a los alumnos, si alguna vez han vivido una experiencia similar y, en ese caso, cuales fueron los elementos más afectados por las sacudidas (botellas llenas o vacías, vasos, platos, etc.). Puede relacionarse la forma, el peso e incluso la posición del centro de gravedad de cada objeto con su estabilidad frente al paso de la onda.

Análisis de la experiencia y vinculación al proceso real: Aunque la experiencia tiene mayor vinculación con los efectos que con las causas de las ondas superficiales permite visualizar que las ondulaciones macroscópicas de la superficie terrestre son las mayores responsables de la destrucción que genera un sismo. Será fácil ver, además, que ha sido más fácil volcar botellas vacías que llenas y más fácil hacerlo con éstas que con los vasos y que es difícil desplazar de su lugar los platos, aún cuando su contenido pueda salir proyectado fuera de ellos, con las consiguientes implicancias para la reflexión acerca de la prevención antisísmica.

Aspectos que son reproducidos aceptablemente: el carácter complejo y destructivo de las ondas superficiales.

Aspectos en los que el modelo no es fidedigno: no se reproducen las verdaderas trayectorias de las partículas que son alcanzadas por las ondas superficiales ni los diferentes tipos de ondas superficiales existentes.

\section{Consideraciones finales}

Las propuestas presentadas pueden ser completadas con otras de acuerdo a la disponibilidad de tiempo y el interés de los estudiantes en el tema. Permanentemente se publican experiencias y propuestas en los sitios vinculados a la enseñanza de las ciencias de la Tierra. Una importante fuente de ejemplos, de mucho interés y poco costo, se encuentra en el sitio de Earth Learning Idea, con traducciones a diferentes idiomas. Algunas de ellas ya se han mencionado, pero existen otras como " $U n$ terremoto tras la ventana, ¿qué verías? ¿qué sentirías?" que aborda la posibilidad de indagar en los conocimientos de los alumnos con respecto a los terremotos y su impacto en un ámbito urbano. Recurrir a la actividad "Sobrevivir al terremoto. Conoce las reglas de comportamiento durante un terremoto e incrementa tus posibilidades de supervivencia" puede contribuir a la 
educación para la prevención y mitigación de los daños generados por estos fenómenos. Geobattleships Do earthquakes and volcanoes coincide? es una variante de la tradicional Batalla Naval que analiza el grado de coincidencia entre las áreas de la Tierra en las que tienen lugar terremotos y fenómenos volcánicos.

González \& Juan (2008) y González et al. (2010) ofrecen muy recomendables propuestas que incluyen la descripción de materiales disponibles en la red para trabajar en la simulación de situaciones catastróficas y actividades de aula a partir de la información disponible en la web y en los medios gráficos. Morcillo Ortega et al. (2006) describe los materiales y resultados del uso de un laboratorio virtual sobre los terremotos a partir de la traducción al español de materiales desarrollados por la United States National Science Fundation y California State University System. Entre los trabajos recientes que utilizan una versión elaborada del dispositivo descripto en la actividad 3 puede mencionarse Peláez et al. (2018), en el cual se brindan referencias a diferentes aproximaciones al uso de la denominada "máquina de hacer terremotos".

\section{Agradecimientos}

El autor agradece a la Dra. Patricia Alvarado por las correcciones y sugerencias realizadas al manuscrito inicial y al equipo responsable y colaboradores de ELI por su desinteresada contribución al mejoramiento de la enseñanza de las Ciencias de la Tierra. A los árbitros de Terree Didatica por sus correcciones y sugerencias para la mejora de esta contribución.

\section{Referencias}

González, M. \& Juan, X. (2008). Aula Virtual: Herramientas de Comunicación. La gestión de los riesgos naturales: Recursos en la red. Enseñanza de las Ciencias de la Tierra, 16 (1), 99-106. URL: https:/www.raco.cat/index.php/ECT/article/ view/120992/166507. Acceso 17-06-2020.

González, M, Alfaro, P. \& Brusi, D. (2010). Recursos para trabajar los terremotos "mediáticos" en el aula. XVI Simposio Enseñanza de la Geología (Teruel), Actas, AEPECT. p. 137-152.

Morcillo Ortega, J.G., García García, E., López García, M. \& Mejías Tirado, N.E. (2006). Los terremotos: Un laboratorio virtual. Su utilización con alumnos de la F. de Educación. XIV Simposio sobre Enseñanza de la Geología (Aveiro), Actas, AEPECT. p. 355-360.

Pantosti, D. (2010). El terremoto esperado pero impredecible. Enseñanza de las Ciencias de la Tierra, 18(3), 333335. URL: https://www.raco.cat/index.php/ECT/article/view/233848/327339. Acceso 17-06-2020.

Peláez, J. A., del Pozo, G. \& García, A. J., 2018. La “máquina" o "generador" de terremotos en un laboratorio universitario. Enseñanza de las Ciencias de la Tierra, 26(2), 186-196. URL: https://www.raco.cat/ index.php/ECT/article/view/338611/429563. Acceso 17-06-2020.

Sellés-Martínez, J. (2005). ¿Qué nos cuentan las ondas sísmicas? Enseñanza de las Ciencias de la Tierra, 13 (1), 20-36. URL: https:/www.raco.cat/index.php/ ECT/article/view/89020/133472\%20. Acceso 1706-2020.

Sellés-Martínez, J. \& Bonán, L. (2000). Problemas de enseñanza-aprendizaje del tema mecanismos focales. Enseñanza de las Ciencias de la Tierra, 8(1), 54-61, URL: https://www.raco.cat/index.php/ECT/article/ view/88654/132728. Acceso 17-06-2020. 